\title{
Characteristics of energy dissipation in hyperconcentrated flows
}

\author{
An-ping $\mathrm{SHU}^{1}$, Qing-quan $\mathrm{LIU}^{2}, \mathrm{Yu}$-jun $\mathrm{YI}^{3}$, and Zhi-dong $\mathrm{ZHANG}^{4}$
}

\begin{abstract}
An equilibrium equation for the turbulence energy in sediment-laden flows was derived on the basis of solid-liquid two-phase flow theory. The equation was simplified for two-dimensional, uniform, steady and fully developed turbulent hyperconcentrated flows. An energy efficiency coefficient of suspended-load motion was obtained from the turbulence energy equation, which is defined as the ratio of the sediment suspension energy to the turbulence energy of the sediment-laden flows. Laboratory experiments were conducted to investigate the characteristics of energy dissipation in hyperconcentrated flows. A total of 115 experimental runs were carried out, comprising 70 runs with natural sediments and 45 runs with cinder powder. Effects of sediment concentration on sediment suspension energy and flow resistance were analyzed and the relation between the energy efficiency coefficient of suspended-load motion and sediment concentration was established on the basis of experimental data. Furthermore, the characteristics of energy dissipation in hyperconcentrated flows were identified and described. It was found that the high sediment concentration does not increase the energy dissipation; on the contrary, it decreases flow resistance.
\end{abstract}

Key Words: Hyperconcentrated flows, Turbulence energy, Efficiency coefficient, Energy dissipation

\section{Introduction}

Hyperconcentrated flow is sediment-laden flow with the concentration exceeding $200 \mathrm{~kg} / \mathrm{m}^{3}$ and a certain amount of fine silt and clay being carried. Hyperconcentrated flow frequently occurs in the Yellow River of China. A number of studies on the characteristics of turbulence and sediment transport in sediment-laden flows were conducted on the basis of stream power theory. Bagnold (1966) first presented the concept of stream power, and analyzed the relation between the energy available and the work done by the flow in transporting sediment. Yang (1976) first presented the theory of minimum stream power and established the equations of unit stream power for total load in 1979 and 1984. Using the theory, Chang (1979) applied the Einstein-Brown and Engelund-Hansen formulas to compute channel geometry and river pattern. Yang's stream power concept is different from that of Bagnold (1966); the former is power per unit bed area and the latter is power per unit weight of water. These two concepts lead to the use of two different and independent parameters relating the sediment transport rate to the energy dissipation rate in open channel flows. Wang et al. $(2004,2007)$ demonstrated how the longitudinal bed profile of the Lower Yellow River can develop into a convex shape in the long run, in contrast to the

'Assoc. Prof., School of Environment, Key Laboratory of Water and Sediment Sciences of Ministry of Education, Beijing Normal University, Beijing 100875, China, E-mail: shuap@bnu.edu.cn.

${ }^{2}$ Prof., Institute of Mechanics, Chinese Academy of Sciences, Beijing 100080, China, E-mail: qqliu@imech.ac.cn.

${ }^{3}$ Lecturer, Dr., School of Environment, Beijing Normal University, Beijing 100875, Cnina, E-mail: yiyujun@bnu.edu.cn.

The project is supported by the National Natural Science Foundation of China (Grant No. 10672024).

${ }^{4}$ Graduate student, School of Environment, Beijing Normal University, Beijing 100875, China, E-mail: gulley@163.com

Note: The original manuscript of this paper was received on Dec. 2007. The revised version was received on July 2008. Discussion open until Dec. 2009. 
concave shapes of all other rivers in the world, which supported the minimum stream power theory developed by Yang $(1976,1987,2002)$. Annandale (2004) studied the rate of sediment transportation and concluded the sediment transport rate is not solely related to the shear stress of flow and stream power. Yang et al. (2007) suggested a method for computing the amount of bed-material load in rivers, which was based on the concept of the modified Bagnold's stream power.

Studies on the laws of energy dissipation have also focused on the energy equilibrium equation. Xie (1957) studied the laws of sediment-laden flows and established a general energy equilibrium equation. Bagnold (1966) first proposed the concepts of sediment suspension energy in the flow and the energy efficiency coefficient for the suspended-load motion $\left(e_{*}\right)$. He studied sediment transport with the energy efficiency coefficient $e_{s}$, which is defined as the ratio of the sediment suspension energy to the turbulence energy of water flow. Qian and Wan (1986) analyzed the relation between the effective sediment suspension energy coefficient and the von Karman's constant. The value of $e_{s}$ varies over a wide range (0.00004-0.20) from laboratory and field data. Wang et al. $(1991,1996)$ concluded that turbulence in hyperconcentrated flows is produced in the turbulent layer; and, high-frequency turbulence is suppressed by the high viscosity and yield stress.

The characteristics of energy dissipation in hyperconcentrated flows are still unclear. According to Shu (1992), the energy efficiency coefficient $e_{s}$ is mainly affected by the flow intensity and sediment concentration. The sediment concentration has a more significant effect on $e_{s}$ in hyperconcentrated flows than in low concentration flows. Fei and Shu (2004) calculated the velocity and resistance for debris flows and hyperconcentrated flows using energy equations.

In this study, the sediment suspension energy $\left(E_{s}\right)$ and the energy efficiency coefficient $\left(e_{s}\right)$ for suspended-load motion are derived from the time-mean turbulence energy equation of solid-liquid two-phase flow. Laboratory experiments are also conducted to verify these equations. Variations in the effective $E_{s}, e_{v}$ and flow resistance coefficient $\left(f_{m}\right)$ with sediment concentration in hyperconcentrated flows are analyzed according to experimental results.

\section{Equilibrium equation for turbulence energy}

Under the hypothesis of a continuous medium, water and sediment in the sediment-laden flows are regarded as the liquid-phase and solid-phase of two-phase flow, respectively. According to the equations of continuity and motion for two-phase flows, an instantaneous energy equation for sediment-laden flows can be written as (Shu, 1994)

$$
\frac{\partial}{\partial t}\left(\rho_{m} B\right)+\frac{\partial}{\partial x_{j}}\left(\rho_{m} B u_{m, j}\right)=-u_{m j} \frac{\partial P_{m}}{\partial x_{i}}+u_{m} \frac{\partial \tau_{m, j}}{\partial x_{j}}+\rho_{m} u_{m, j} g_{m, j}-u_{m, j} \frac{\partial A}{\partial x_{j}}
$$

(I)

(III)

(IV)

(V)

(VI)

where subscript $m$ denotes mixture-phase flow or sediment-laden flow; $\rho_{m}$ and $u_{m}$ are density and velocity of the mixture-phase flow, respectively; $B=\frac{1}{2} u_{m, i}^{2}$ is the total kinetic energy per unit mass for the mixture-phase flow; $A=\frac{\rho \rho_{s}}{\rho_{m}} S_{v}\left(1-S_{v}\right) \omega^{2} \delta_{t 3} \delta_{i 3}$ is the additional resistance due to specific gravity difference between sediment and water phases; $S_{v}$ is the volume sediment concentration; $\omega$ is the fall velocity of sediment particles; $\rho$ and $\rho$, are densities for clear water and sediment, respectively; and $\delta_{i 3}$ is a two-dimensional tensor $(i=1,2,3)$. The terms of Eq. (1) are explained as follows.

(I) $\frac{\partial}{\partial t}\left(\rho_{m} B\right)$ is the variation in the kinetic energy with time; 
(II) $\frac{\partial}{\partial x_{j}}\left(\rho_{m} B u_{m, j}\right)$ is the variation in the kinetic energy with distance;

(III) $u_{m} \frac{\partial P}{\partial x_{i}}$ is the work due to pressure gradient;

(IV) $u_{m, j} \frac{\partial \tau_{m, j}}{\partial x_{j}}$ is the work due to viscous energy dissipation;

(V) $\rho_{m} u_{m} g_{m,}$ is the work due to gravity; and

(VI) $\frac{\partial A}{\partial x_{i}}$ is the work due to the difference between the gravities of sediment and water.

2.1 Time-mean equilibrium equation for turbulence energy

The variables in Eq. (1) can be replaced by the summations of the time-mean value and the fluctuation:

$$
u_{m, i}=\bar{u}_{m, i}+u_{m, i}^{\prime}, \rho_{m}=\bar{\rho}_{m}+\rho_{m}^{\prime}, \quad P_{m}=\bar{P}_{m}+P_{m}^{\prime}, \tau_{m, i j}=\bar{\tau}_{m, i j}+\tau_{m, i j}^{\prime} \text {, and } A=\bar{A}+A^{\prime}
$$

After averaging over time, the time-mean equilibrium equation for turbulence energy of mixture-phase flow is

$$
\begin{aligned}
& \frac{\partial}{\partial t} \overline{\rho_{m} B_{t}}+\frac{\partial}{\partial x_{j}}\left(\rho_{m} u_{m, j} B_{i}\right)=-\frac{\partial}{\partial x_{j}}\left(\overline{\rho_{m} u_{m, j}^{\prime}} B_{t}+\overline{P_{m}^{\prime} u_{m, j}^{\prime}}\right)-\bar{\rho}_{m} \overline{u_{m, i}^{\prime} u_{m, j}^{\prime}} \frac{\partial \bar{u}_{m, i}}{\partial x_{j}} \\
& +\overline{u_{m, i}^{\prime} \frac{\partial \tau_{m, j}^{\prime}}{\partial x_{j}}}+\overline{\rho_{m}^{\prime} u_{m, i}^{\prime} g_{m, i}}-\overline{u_{m, i}^{\prime} \frac{\partial A^{\prime}}{\partial x_{i}}}
\end{aligned}
$$

where $B_{t}=\frac{1}{2} \overline{u_{m, i}^{\prime 2}}$ is turbulence energy per unit mass for mixture-phase flow.

Some terms in Eq. (2) can be re-written by considering the turbulent shearing stress,

$$
\tau_{m, u}^{\prime}=\mu_{m} \frac{\partial u_{m, j}^{\prime}}{\partial x_{j}}
$$

therefore,

$$
\begin{aligned}
& \overline{u_{m, d}^{\prime} \frac{\partial \tau_{m, 4}^{\prime}}{\partial x_{j}}}=\frac{\partial}{\partial x_{j}} \overline{u_{m, l}^{\prime} \tau_{m, y}^{\prime}}-\overline{\tau_{m, l j}^{\prime} \frac{\partial u_{m, l}^{\prime}}{\partial x_{j}}}, \\
& =\mu_{m} \frac{\partial^{2}}{\partial x_{l}^{2}}\left(\overline{\frac{u_{m, j}^{\prime 2}}{2}}\right)-\mu_{m} \overline{\left(\frac{\partial u_{m, J}^{\prime}}{\partial x_{j}}\right)^{2}}
\end{aligned}
$$

where $\mu_{m}$ is the viscous coefficient for mixture-phase flow.

According to Prandtl's mixture length theory, the diffusion term of turbulence energy can be expressed as

$$
\frac{\partial}{\partial x_{j}}\left[\overline{\rho_{m} u_{m, j}^{\prime}\left(\frac{u_{m, j}^{\prime 2}}{2}\right)}+\overline{P_{m}^{\prime} u_{m, j}^{\prime}}-\mu_{m} \frac{\partial}{\partial x_{j}}\left(\overline{\frac{u_{m, j}^{\prime}}{2}}\right)\right]=\frac{\partial}{\partial x_{j}}\left(\frac{\mu_{e}}{\sigma_{B t}} \frac{\partial B_{i}}{\partial x_{j}}\right) .
$$

Accordingly, the following time-mean equation for the turbulence energy of mixture-phase flow can be obtained:

$$
\frac{\partial}{\partial t}\left(\bar{\rho}_{m} B_{t}\right)+\frac{\partial}{\partial x_{j}}\left(\bar{\rho}_{m} \bar{u}_{m, j} B_{t}\right)=-\frac{\partial}{\partial x_{j}}\left(\frac{\mu_{e}}{\sigma_{B t}} \frac{\partial B_{t}}{\partial x_{j}}\right)-\bar{\rho}_{m} \overline{u_{m, i}^{\prime} u_{m, j}^{\prime}} \frac{\partial \bar{u}_{m, i}}{\partial x_{j}}+\overline{\rho_{m}^{\prime} u_{m, i}^{\prime}} g_{m, l}-\mu_{m} \overline{\left(\frac{\partial u_{m, i}^{\prime}}{\partial x_{j}}\right)^{2}} \overline{-u_{m, i}^{\prime} \frac{\partial A^{\prime}}{\partial x_{j}}}
$$

(I)

(III)

(IV)

(V)

where $\mu_{e}$ is the effective viscosity and consists of the turbulent viscous coefficient $\mu_{T}$ and dynamic viscosity $\mu_{m}$; i.e., $\mu_{e}=\mu_{m}+\mu_{T} \cdot \sigma_{B t}$ is Prandtl's number for the mixture-phase flow. In Eq. (6), 
(I) $\frac{\partial}{\partial t}\left({\widetilde{\rho_{m}}}_{B_{1}}\right)$ is the variation in turbulence energy with time;

(II) $\frac{\partial}{\partial x_{j}}\left(\bar{\rho}_{m} \bar{u}_{m, j} B_{\imath}\right)$ is the variation in turbulence energy with distance;

(III) $\frac{\partial}{\partial x_{j}}\left(\frac{\mu_{e}}{\sigma_{B t}} \frac{\partial B_{t}}{\partial x_{j}}\right)$ is the diffusion term for turbulence energy;

(IV) $\bar{\rho}_{m} \bar{u}_{m, j}^{\prime} u_{m, j}^{\prime} \frac{\partial \bar{u}_{m, l}}{\partial x_{j}}$ is the partial turbulence energy due to the interaction between the turbulent shearing stress and mean velocity gradients in unit time;

(V) $\overline{\rho_{m}^{\prime} u_{m, i}^{\prime}} g_{m, i}$ is the component of turbulence energy dissipated in sediment suspension; i.e., sediment suspension energy;

(VI) $\mu_{m} \overline{\left(\frac{\partial u_{m, i}^{\prime}}{\partial x_{j}}\right)^{2}}$ is the work due to the viscous dissipation of turbulence energy; and

(VII) $\overline{u_{m,}^{\prime} \frac{\partial A^{\prime}}{\partial x_{j}}}$ is the work due to the resistance difference in the relative fluctuations of sediment and water; the term may be ignored in the case of fully turbulent flows.

\subsection{Simplification of the turbulence energy equation for hyperconcentrated flows}

For two-dimensional, uniform, steady and fully developed turbulent hyperconcentrated flows, as shown in Fig. 1, the turbulence energy equation can be simplified on the basis of the following assumptions.

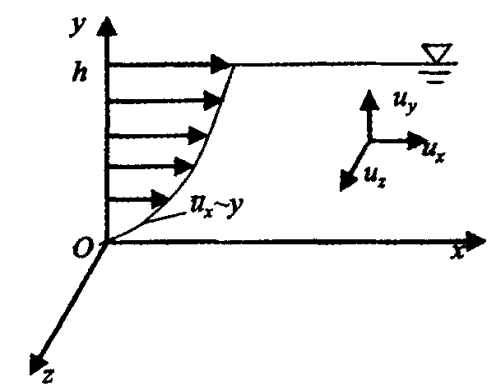

Fig. 1 Sketch diagram of flowing water

(i) For two-dimension flow: $\mu_{z}=0$ and $\frac{\partial}{\partial z}=0$.

(ii) For steady flow: $\frac{\partial}{\partial t}=0$.

(iii) For uniform flow: $\frac{\partial}{\partial x}=0$ and $u_{y}=0$.

(iv) For fully developed turbulent hyperconcentrated flow: $\overline{u_{m,}^{\prime} \frac{\partial A^{\prime}}{\partial x_{j}}}=0$.

(v) For unit mass gravity: $g_{m, y}=-g$ and $g_{m, x}=g_{m, z}=0$.

Eq. (6) is simplified as

$$
-\bar{\rho}_{m} \overline{u_{x}^{\prime} u_{y}^{\prime}} \frac{\partial \bar{u}_{x}}{\partial y}=\frac{\partial}{\partial y}\left(\frac{\mu_{e}}{\sigma_{B}} \frac{\partial B_{i}}{\partial y}\right)++\mu_{m} \overline{\left(\frac{\partial u_{x}^{\prime}}{\partial y}\right)^{2}}+\overline{\rho_{m}^{\prime} u_{y}^{\prime} g} .
$$

For convenience, Eq. (7) may also be written as 


$$
E_{1}=E_{2}+E_{3}+E_{s}
$$

where

$$
\begin{aligned}
& E_{1}=-\rho_{m} \overline{u_{x}^{\prime} u_{y}^{\prime}} \frac{\partial \overline{u_{x}}}{\partial y} \text { is the total turbulence energy; } \\
& E_{2}=\frac{\partial}{\partial y}\left(\frac{\mu_{e}}{\sigma_{s}} \cdot \frac{\partial B_{t}}{\partial y}\right) \text { is the diffusion term of } E_{1} \text {; } \\
& E_{3}=\mu_{m}\left(\frac{\partial u_{x}^{\prime}}{\partial y}\right)^{2} \text { is the viscous dissipation term of } E_{1} \text {; and } \\
& E_{s}=\overline{\rho_{m}^{\prime} u_{y}^{\prime} g} \text { is the sediment suspension energy. }
\end{aligned}
$$

As shown in Eq. (7), the total turbulence energy equals the summation of the diffusion of turbulence energy, viscous dissipation of turbulence energy and sediment suspension energy. In other words, sediment suspension energy comes from turbulence energy in sediment-laden flows.

Furthermore, the production term and dissipation term for turbulence energy may be expressed respectively as

$$
\begin{aligned}
& E_{1}=-\rho_{m} \overline{u_{x}^{\prime} u_{y}^{\prime}} \frac{\partial \overline{u_{x}}}{\partial y}=\rho_{m} l_{m}^{2}\left(\frac{\partial \overline{u_{x}}}{\partial y}\right)^{3}=\rho_{m}\left(\overline{u_{y}^{2}}\right)^{\frac{3}{2}} / l_{m}=4.1 \frac{\rho_{m} u_{*}^{3} \eta_{s}^{3}}{\kappa^{2} h}\left(1-\frac{y}{h}\right)^{\frac{3}{2}} \text { and } \\
& E_{3}=\mu_{m}\left(\frac{\partial u_{x}^{\prime}}{\partial y}\right)^{2}=C_{d} \mu_{T} \frac{2 B_{t}}{l_{m}}=C_{\mu} C_{d}\left(1+C_{1}^{2}\right)^{\frac{3}{2}} \rho_{m}{\overline{\left(u_{y}^{2}\right.}}^{\frac{3}{2}} / l_{m}=C_{\mu} C_{d}\left(1+C_{1}^{2}\right)^{\frac{3}{2}} \cdot E_{1}
\end{aligned}
$$

where $C_{\mu}$ is the correction coefficient; $C_{d}$ is the proportional coefficient for the turbulence energy dissipated in the flow, which is related to the turbulence exchanging factor; $C_{1}$ is the ratio of turbulence intensities in the transverse and longitudinal directions; and $\eta_{s}$ is a factor reflecting the effect of sediment suspension on the vertical turbulence intensity of sediment-laden flows.

\subsection{Sediment suspension energy}

Substituting Eqs. (13) and (14) into Eq. (8), a differential equation for the energy equilibrium is obtained as

$$
E_{1} \cdot\left[1-C_{\mu} C_{d}\left(1+C_{1}^{2}\right)^{\frac{3}{2}}\right]=\frac{\partial}{\partial y}\left(\frac{\mu_{e}}{\sigma_{B t}} \frac{\partial B_{t}}{\partial y}\right)+E_{s} .
$$

By integrating Eq. (15) over the water depth $h$, a depth-averaged turbulence energy equation is obtained:

$$
\gamma_{m} \Phi \frac{\sqrt{f_{m}} U J}{\kappa^{2}} \int_{a}^{h}\left(1-\frac{y}{h}\right)^{\frac{3}{2}} d y=\int_{a}^{h} E_{s} d y=\overline{E_{s}}(h-a)
$$

Thus,

$$
\bar{E}_{s}=\gamma_{m} \Phi \frac{\sqrt{f_{m}} U J}{\kappa^{2}}\left(1-\frac{a}{h}\right)^{\frac{3}{2}} \approx \gamma_{m} \Phi \frac{\sqrt{f_{m}} U J}{\kappa^{2}} \quad(a \ll h),
$$

where $\Phi$ is a comprehensive index.

$$
\Phi=1.64\left[1-C_{\mu} C_{d}\left(1+C_{1}^{2}\right)^{\frac{3}{2}}\right] \eta_{s}^{3},
$$

where $\overline{E_{s}}$ is the depth-averaged work due to sediment suspension (i.e., sediment suspension energy); International Journal of Sediment Research, Vol. 23, No. 4, 2008, pp. 387-397 
$a$ is the thickness of the bed-load layer; and $a=2 D_{65}$, where $D_{65}$ is the grain diameter for which $65 \%$ of grains are finer).

2.4 Energy efficiency coefficient for suspended-load motion

For sediment-laden turbulent flows, an energy efficiency coefficient $e_{8}$ is defined as the ratio of the sediment suspension energy, $E_{\mathrm{s}}$, to the turbulence energy, $E_{l}$ :

$$
e_{s}=\frac{E_{s}}{E_{1}}=\frac{\overline{\rho_{m}^{\prime} v^{\prime} g}}{-\rho_{m} \overline{u^{\prime} v^{\prime}} \frac{\partial u}{\partial y}}
$$

Integrating $E_{\mathrm{s}}$ and $E_{l}$ over the flow depth gives the following depth-averaged expressions.

and

$$
\overline{E_{s}}=\frac{1}{h-a} \int_{a}^{h} \rho_{m}^{\prime} \nu^{\prime} g d y=\frac{1}{h-a} \int_{a}^{h}\left(\gamma_{s}-\gamma_{m}\right) S_{v} \omega d y=\left(\gamma_{s}-\gamma_{m}\right) \overline{S_{v}} \cdot \bar{\omega}
$$

$$
\overline{E_{1}}=\frac{1}{h-a} \int_{a}^{h} \tau^{\prime} \frac{\partial u}{\partial y} d y=\frac{1}{h-a} k_{t} \cdot \int_{a}^{h} \tau \frac{\partial u}{\partial y} d y=k_{t} \gamma_{m} U J
$$

Therefore, $e_{s}$ is obtained for sediment-laden flows with a large amount of suspended-load:

$$
e_{s}=\frac{\overline{E_{s}}}{\overline{E_{1}}}=\frac{\left(\gamma_{s}-\gamma_{m}\right) S_{v} \omega}{k_{t} \gamma_{m} U J},
$$

where $k_{t}$ is the transfer ratio for turbulence energy in the two-phase mixture flow, defined as the ratio of the depth-averaged values of turbulence energy to effective potential energy, expressed as

$$
k_{t}=1-\frac{1}{\tau_{0} h}\left[\tau_{B T}\left(2 h-y_{0}\right)+2 \mu_{m} k_{d} U\right] \text {, }
$$

where $y_{0}=\frac{\tau_{B T}}{\tau_{0}} h$ is the thickness of the flowing core in hyperconcentrated flows; $u_{\max }=k_{d} U$ is the maximum flow velocity over the vertical; $k_{d}$ is a shape coefficient for the velocity profile over depth $\left(k_{d}>1\right)$; and $U$ is the depth-averaged velocity of water flows. Eq. (22) can be rewritten as

$$
\overline{E_{s}}=e_{s} \overline{E_{1}}=e_{s} k_{t} \gamma_{m} U J \text {, }
$$

where $\gamma_{s}$ and $\gamma_{m}$ are the specific gravities for solid and mixture phases, respectively and $J$ is the hydraulic gradient.

Substituting Eq. (24) into Eq. (17) gives the final expression for $e_{s}$ as

$$
e_{s}=\Phi \frac{1}{\kappa^{2} k_{t}}\left(\frac{f_{m}}{8}\right)^{\frac{1}{2}},
$$

where $\kappa$ is the von Karman constant and $f_{m}$ is the resistance coefficient for sediment-laden flows.

According to Fei (1990), the resistance coefficient $f_{m}$ for sediment-laden flows can be expressed as

$$
f_{m}=0.11 \alpha\left(\frac{k_{s}}{4 R}+\frac{68}{R_{e m}}\right)^{0.25},
$$

where $\alpha$ is a reducing resistance coefficient less than $1 ; R$ is the hydraulic radius; $k_{s}$ is the roughness of the river bed; and $R_{e m}$ is Reynold's number for hyperconcentrated flows.

In addition, the comprehensive index $\Phi$ can be obtained as (Shu, 2008)

$$
\Phi=p \frac{\left[\lg \left(\mu_{r}+0.1\right)\right]^{N}}{T^{1-N}}
$$

Here, $\mu_{r}$ is the ratio of the viscosities of sediment-laden flow to clear water; the coefficient $p$ and exponent $N$ are determined using experimental data; and the comprehensive factor for sediment -392 International Journal of Sediment Research, Vol. 23, No. 4, 2008, pp. 387-397 
transportation, $T$, can be expressed as

$$
T=\frac{1}{\kappa^{2}}\left(\frac{f_{m}}{8}\right)^{\frac{3}{2}} \cdot \frac{\gamma_{m}}{\gamma_{s}-\gamma_{m}} \cdot \frac{U^{3}}{g R \omega} .
$$

\section{Laboratory experiments}

\subsection{Experimental setups}

To study the energy dissipation by sediment suspension and transportation in hyperconcentrated flows, two experiments for sediment-laden flows were conducted using flumes. One experiment was carried out in a flume $16 \mathrm{~m}$ long, $0.5 \mathrm{~m}$ wide and $0.5 \mathrm{~m}$ high with a tilting bed slope varying from $0 \%$ to $2.5 \%$ and natural sediment from the Yongding River in the northern of China. The second was conducted in a flume $25 \mathrm{~m}$ long, $0.5 \mathrm{~m}$ wide and $0.6 \mathrm{~m}$ high with cinder powder. The experimental setups are shown in Fig. 2 .

Water depths were measured with an ultrasonic water level gauge. The velocity profile was measured using a Pitot tube and pressure sensor, as shown in Fig. 3. This instrument developed by Shu and Ren (2007) is able to measure flow velocity in hyperconcentrated flows.

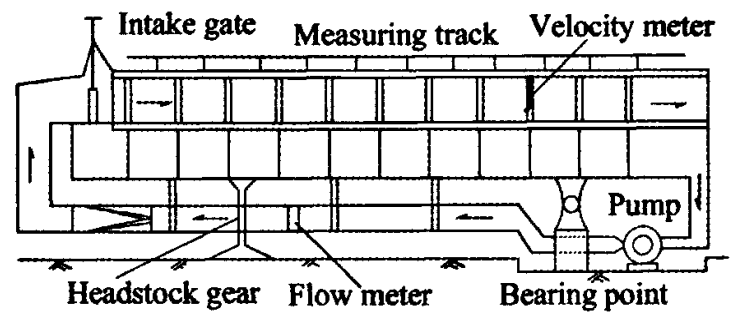

(a) Natural sediment setup

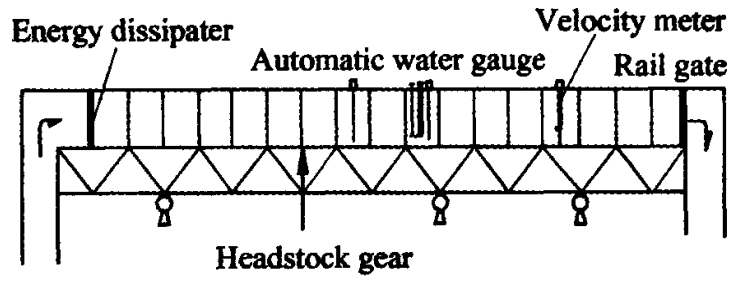

(b) Cinder powder setup

Fig. 2 Experimental setups

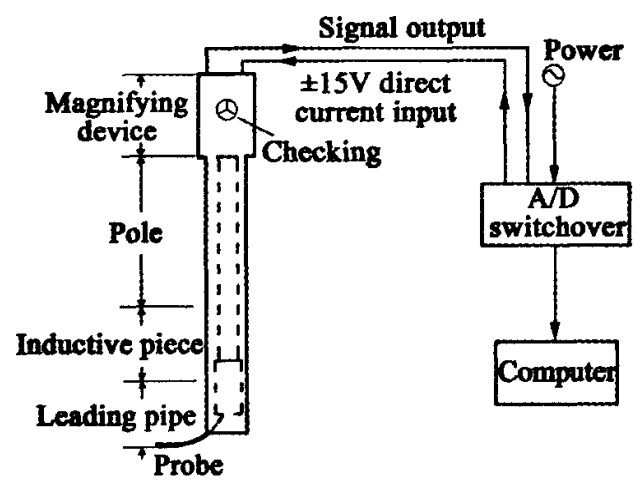

Fig. 3 Sketch diagram of the velocity meter

\subsection{Experimental conditions and methods}

The sediment from the Yongding River has a grain size of $d=0.0016 \mathrm{~mm}-0.4 \mathrm{~mm}$ and median diameter of $d_{s 0}=0.076 \mathrm{~mm}$. The cinder powder has a grain size of $d=0.0009 \mathrm{~mm}-0.2 \mathrm{~mm}$ and a median diameter of $d_{50}=0.017 \mathrm{~mm}$. The size distributions of the two materials are shown in Fig. 4. Experimental conditions are summarized in Table 1.

The sediment was spread to a $20 \mathrm{~cm}$ thickness on the flume bottom before each run in the natural sediment experiments. Clear water was then poured into the flume and the sediment layer was scoured away with increasing discharge. When a stable equilibrium state for sediment transport was established, several important hydraulic and sediment factors, such as velocity, water depth, hydraulic gradient and sediment concentration, were measured.

For the experiments with cinder powder, cinder power suspension flowed into the flume from a tank in 
which the cinder powder was fully mixed with water. Initially, the cinder powder partially deposited on the flume bottom. The sediment-laden flows reached an equilibrium state when the deposition stopped.

A total of 70 natural sediment runs and 45 cinder powder runs were conducted in the laboratory, and important parameters, such as discharge, velocity, hydraulic gradient, sediment concentration, hydraulic gradient and grain size, were measured. The experimental data were used to study energy dissipation and sediment transport in hyperconcentrated flows.

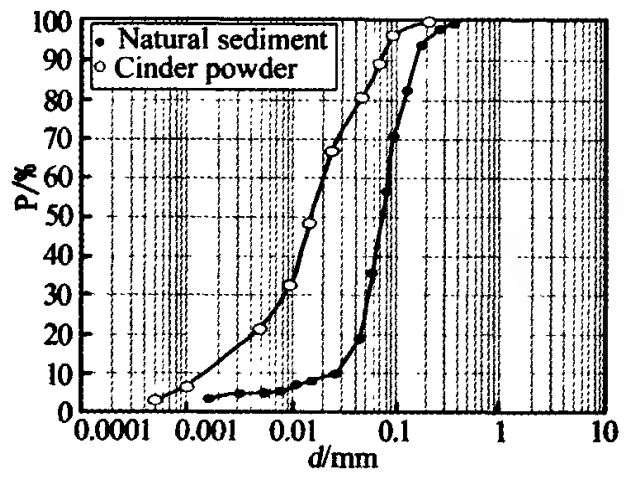

Fig. 4 Grain size distributions of the experimental sediments

Table 1 Experimental Conditions

\begin{tabular}{|c|c|c|c|c|c|c|}
\hline $\begin{array}{c}\text { Experimental } \\
\text { types }\end{array}$ & $\begin{array}{c}\text { Specific } \\
\text { gravity } \\
\gamma_{s} / t \cdot \mathrm{m}^{3}\end{array}$ & $\begin{array}{c}\text { Grain } \\
\text { diameter } \\
d_{s 0} / \mathrm{mm}\end{array}$ & $\begin{array}{c}\text { Concentration } \\
S / \mathrm{kg} \cdot \mathrm{m}^{3}\end{array}$ & $\begin{array}{c}\text { Discharge } \\
Q / 1 \cdot \mathrm{s}^{1}\end{array}$ & $\begin{array}{c}\text { Flow } \\
\text { gradient } \\
J / \% \text { t }\end{array}$ & $\begin{array}{c}\text { Water } \\
\text { depth } \\
h / \mathrm{cm}\end{array}$ \\
\hline $\begin{array}{c}\text { Natural } \\
\text { sediment }\end{array}$ & 2.65 & 0.076 & $2.61-765.61$ & $9.06-94.87$ & $0.17-5.40$ & $7.0-17.2$ \\
\hline Cinder powder & 2.17 & 0.017 & $3.10-539.70$ & $12.50-55.00$ & $0.26-3.13$ & $5.5-15.0$ \\
\hline
\end{tabular}

\section{Results analysis}

4.1 Relationship between sediment suspension energy and sediment concentration

The coefficient $p$ and exponent $N$ in Eq. (27) were determined as 0.3551 and 0.72 , respectively, using experimental data. Thus the comprehensive index $\Phi$ can be determined as

$$
\Phi=0.3551 \frac{\left[\lg \left(\mu_{r}+0.1\right)\right]^{0.72}}{T^{0.28}} \text {. }
$$

Substituting Eq. (29) into Eq. (17), the sediment suspension energy $E$, can be expressed as

$$
\overline{E_{s}}=0.3551\left[\lg \left(\mu_{r}+0.1\right)\right]^{0.72} \frac{\sqrt{f_{m}}}{\kappa^{2} \cdot T^{0.28}} \cdot \gamma_{m} U J \text {. }
$$

Relations between sediment suspension energy $E_{s}$, calculated using Eq. (30), and sediment concentration $S_{v}$ were also obtained using experimental data (Fig. 5). It can be seen the relations of the sediment suspension energy $E_{s}$ and sediment concentration $S_{v}$ for the two different types of sediment transport experiments were similar. $E$, increased with increasing $S_{v}$ when $S_{v}$ was less than 0.15 ( $S \approx 400 \mathrm{~kg} / \mathrm{m}^{3}$ for natural sediment and $S \approx 320 \mathrm{~kg} / \mathrm{m}^{3}$ for cinder powder), whereas $E_{s}$ decreased as $S_{v}$ increased in hyperconcentrated flows with $S_{v}>0.15$. In other words, $E$, reached a maximum when $S_{v}$ was about 0.15 . The decrease in the energy dissipated in maintaining the suspended-load motion was due to the decrease in the resistance to hyperconcentrated flows. The relation between $E_{s}$ and $S_{v}$ basically agrees with the relation between $e_{s}$ and $S_{v}$. These results explain why hyperconcentrated flows can carry such a huge amount of fine sediment through the Yellow River without a large hydraulic gradient. 


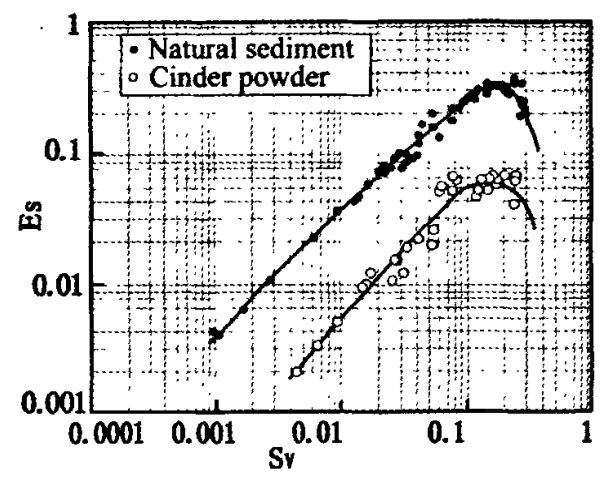

Fig. 5 Relations of $E_{s}$ and $S_{v}$

4.2 Variations of the energy efficiency coefficient with sediment concentration

Substituting Eq. (29) into Eq. (25), the energy efficiency coefficient of the suspended-load motion is obtained:

$$
e_{s}=0.3551 \frac{\left[\lg \left(\mu_{r}+0.1\right)\right]^{0.72}}{\kappa^{2} k_{t} T^{0.28}}\left(\frac{f_{m}}{8}\right)^{\frac{1}{2}} .
$$

From Eq. (31), the value of $e_{s}$ can be calculated using experimental data, and the resulting relation of $e_{s}$ and $S_{v}$ is shown in Fig. 6. For low sediment concentration flows $\left(S_{v}<0.02\right)$, the value of $e_{s}$ is basically constant between 0.006 and 0.01 , which confirms the experimental result of $\left(1-e_{b}\right) e_{s}=0.01$ presented by Bagnold (1966). As $S_{v}$ increased, the value of $e_{s}$ also increased, which indicates $S_{v}$ had a dominant effect on $e_{s}$ in flows with a mid-range sediment concentration $\left(0.02<S_{v}<0.15\right)$. For hyperconcentrated flows $\left(S_{v}>0.15\right)$ with either natural sediments or cinder particles, the value of $e_{\text {, }}$ decreased obviously as $S_{v}$ increased, which clearly suggests the increase in sediment concentration does not require an increase in energy expenditure on suspended-load motion in byperconcentrated flows.

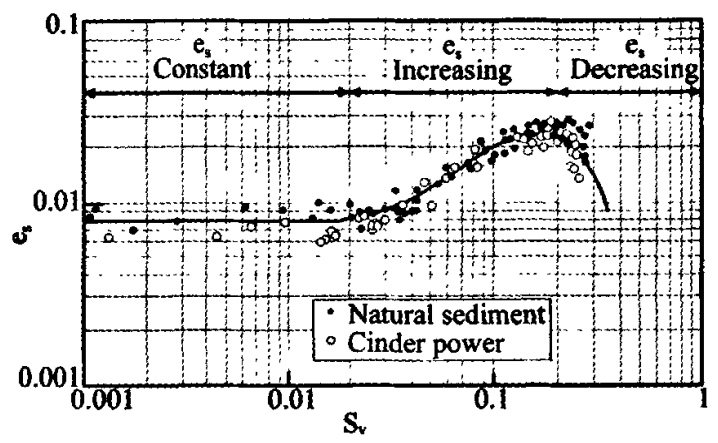

Fig. 6 Variations in $e_{s}$ with $S_{v}$

For hyperconcentrated flows, the relations between $E_{s}$ and $S_{v}$ are similar in the experiments for natural sediment and cinder powder. Therefore, the energy efficiency coefficient $e_{x}$ was closely related to the sediment concentration $S_{v}$ and was basically independent of the specific gravity.

4.3 Relations between the flow resistance coefficient and sediment concentration

The relations between the flow resistance coefficient $\left(f_{m}\right)$, energy efficiency coefficient $\left(e_{s}\right)$, and sediment concentration $\left(S_{v}\right)$ are shown in Fig. 7, in which $f_{m}$ is calculated using Eq. (26). The results demonstrate $f_{m}$ generally decreases with increasing $S_{v}$ and energy efficiency coefficient $e_{s}$. For 
hyperconcentrated flows with a concentration $S_{v}>0.15$, the flow resistance coefficient $f_{m}$ decreases with increasing $S_{v}$, but varies within the range of $f_{m}=0.0018-0.0025$, where $e_{s}$ is in the range $0.02-0.03$. It is shown the flow resistance in hyperconcentrated flows is smaller than that in low-concentration flows.
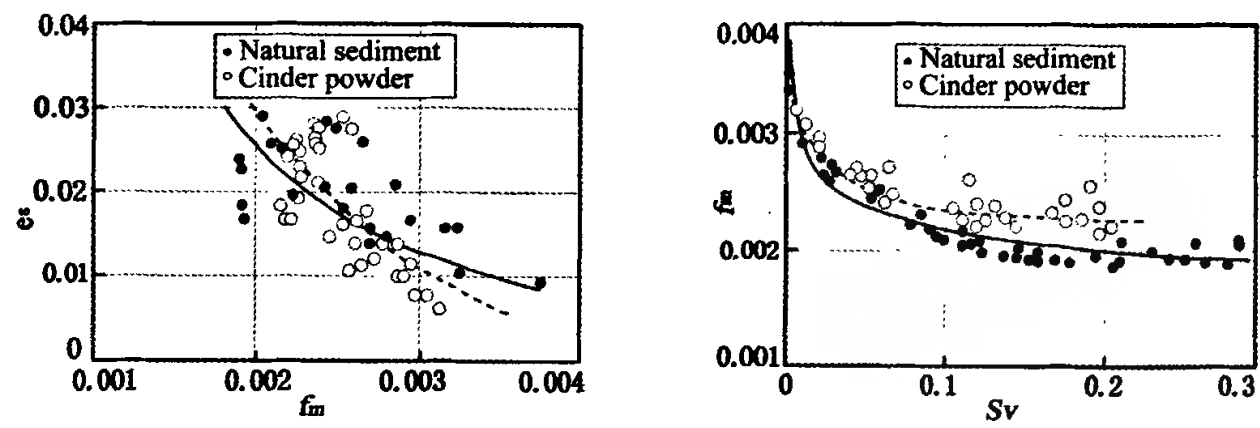

Fig. 7 Relations between $e_{s}$ and $f_{m}$, and $f_{m}$ and $S_{v}$

\section{Conclusions}

A time-mean turbulence energy equation for sediment-laden flows is presented in this study and the equation is simplified for two-dimensional, uniform, steady and fully-developed turbulent hyperconcentrated flows. The sediment suspension energy and the energy efficiency coefficient of suspended-load motion were derived.

Relations between sediment suspension energy, the energy efficiency coefficient, and the volume sediment concentration were obtained from laboratory experiments. The value of the energy efficiency coefficient is constant in the case of low-concentration sediment-laden flows, and increases as the sediment concentration increases in the transitional range from low to high concentrations. It deceases with a further increasing in the concentration for hyperconcentrated flows. The flow resistance also decreases with increasing sediment concentration in hyperconcentrated flows. The relation between the energy efficiency coefficient and sediment concentration is normally similar to the relation of the sediment suspension energy and sediment concentration. This suggests that owing to a decrease in flow resistance, the energy dissipated in the suspended-load motion decreases when the sediment concentration increases in hyperconcentrated flows.

\section{Acknowledgements}

The flume experiments for sediment transport were carried out at the State Key Laboratory of Hydroscience and Engineering, Tsinghua University, and the Yellow River Institute of Hydraulic Research. Support from experts at these laboratories is greatly appreciated. This study is supported by the National Natural Science Foundation of China (Grant No. 10672024 and No.10825211).

\section{References}

Annandale G. W. 2004, Erosive capacity, resistance and process. 2nd International Conference on Scour and Erosion, Singapore, No. 1, pp. 1-20.

Bagnold R. A. 1966, An approach to the sediment transport problem from general physics, U.S. Geo. Sur., Prof. Paper 422-I, 422.

Chang H. H. 1979, Minimum stream power and river pattern. Journal of Hydrology, 41, pp. 303-307.

Fei X. J. 1990, Research on turbulence resistance for pseudo-homogeneous flow. Journal of Hydraulic Engineering, No. 12, pp. 48-54 (in Chinese).

Fei X. J. and Shu A. P. 2004, Movement mechanism and disaster control for debris flow. Tsinghua University Press, 144-159 (in Chinese).

Qian N. and Wan S. H. 1986, Mechanics of sediment Motion. Science Press, pp. 346-363 (in Chinese).

Shu A. P. 1992, A preliminary study on the energy efficiency coefficient of sediment suspension motion. Journal of Wuhan University of Hydraulic and Electric Power, Vol. 25, No. S1, pp. 133-138 (in Chinese).

Shu A. P. 1994, Study on the sediment transport capacity and mechanism of hyperconcentrated flow. Doctoral Thesis of Tsinghua University, pp. 3-12, 35-42, 90-94 (in Chinese). 
Shu A. P. and Fei X. J. 2008, Sediment transport capacity of hyperconcentrated flow. Science in China Series G: Physics, Mechanics and Astronomy, Vol. 51, No. 8, pp. 961-975.

Shu A. P. and Ren Y. M. 2007, Development and application of a new velocity meter for hyperconcentrated flow. Journal of Sediment Research, No. 4, pp. 59-64 (in Chinese).

Wang Z. Y. and Erich J. Plate. 1996, A preliminary study on the turbulence structure of flows of non-Newtonian fluid. Journal of Hydraulic Research, Vol. 34, No. 3, pp. 345-361.

Wang Z. Y. and Hu C. H. 2004, Interactions between fluvial systems and large-scale hydro-projects. Keynote Lecture at 9th International Symposium on River Sedimentation, Yichang China.

Wang Z. Y. and Zhang X. Y. 1991, Effects of bed load motion on turbulence structure of water flow and bingham mud flow. Journal of Sediment Research, No. 2, pp. 14-28 (in Chinese).

Wang Z. Y., Wu B. S., and Wang G. Q. 2007, Fluvial processes and morphological response in the Yellow and Weihe Rivers to closure and operation of Sanmenxia Dam. Geomorphology, Vol. 91, No. 1-2, pp. 65-79.

Xie J. H. 1957, Energy equilibrium problems about carrying capacity for sediment-laden flows, Journal of Sediment Research, No. 2, pp. 35-65 (in Chinese).

Yang C. T. 1976, Minimum unit stream power and fluvial hydraulics. Journal of Hydraulic Division, ASCE, Vol. 102, No. HY07, pp. 919-934.

Yang C. T. 1979, Unit stream power equations for total load. Joumal of Hydrology, 40, pp. 123-138.

Yang C. T. 1984, Unit stream power equation for gravel. Journal of Hydraulic Engineering, ASCE, Vol. 110, No. HY12, pp. 1783-1797.

Yang C. T. and Song C. C. S. 1987, Theory of minimum energy and energy dissipation rate. Journal of Hydraulic Engineering, ASCE, Vol. 113, No. HY07, pp. 769-784.

Yang C. T. 2002, Sediment transport and stream power. International Journal of Sediment Research, Vol. 17, No. 1, pp. 31-38.

Yang S. Q., Koh S. C., Kim I. S., and Song Y. C. 2007, Sediment transport capacity -an improved Bagnold's formula. International Journal of Sediment Research, Vol. 22, No. 1, pp. 27-38. 